\title{
Impingement, coalescence and mixing of micro-droplets on a solid surface
}

\author{
Guina $\mathrm{Yi}^{1}$, Ziqi $\mathrm{Cai}^{2}$, Zhengming $\mathrm{GaO}^{2}$, and Jos Derksen ${ }^{3}$ \\ ${ }^{1}$ Beijing University of Chemical Technology \\ ${ }^{2}$ Affiliation not available \\ ${ }^{3}$ University of Aberdeen
}

May 22, 2021

\begin{abstract}
The coalescence of an impinging droplet colliding with a sessile droplet at an angle $(\vartheta \mathrm{i})$ is investigated by numerical simulation. The range of $\vartheta \mathrm{i}$ is $0^{\circ}-60^{\circ}$ and the surface wettability are set as hydrophilic or hydrophobic, and both of them can affect the droplet mergence behavior. By using a modified mixing function, the dimensionless total mixing time $\tau \mathrm{m}$ can be calculated. The results show that there is no clear effect of $\vartheta \mathrm{i}$ on $\tau \mathrm{m}$ on a hydrophobic surface, while $\tau \mathrm{m}$ increases as $\vartheta \mathrm{i}$ increases on the hydrophilic surface. With the Weber number(We) ranging from 5.65 to 22.7 and the Ohnesorge number(Oh) ranging from 0.136 to 0.214 , we find $\tau \mathrm{m}$ hardly changes with We and Oh. By dividing the mergence and mixing process in a convection and a diffusion stage, we find that the diffusion is much larger than the convection time.
\end{abstract}

\section{Hosted file}

DoubleDropletsManuscript20210507_FinalVersion_plaintxt.docx available at https://authorea. com/users/415190/articles/523091-impingement-coalescence-and-mixing-of-micro-dropletson-a-solid-surface 
Table 1. Parameters settings in MDPD simulations

\begin{tabular}{|c|c|c|}
\hline description & MDPD units & Physical units \\
\hline Radius, $R_{\mathrm{c}}$ & 14 & $18.5 \mu \mathrm{m}$ \\
\hline density, $\rho$ & 6.09 & $1056 \mathrm{~kg} / \mathrm{m}^{3}$ \\
\hline Surface tension, $\sigma$ & 7.51 & $56.5 \mathrm{mN} / \mathrm{m}$ \\
\hline dynamic viscosity, $\mu$ & 4.872 & 0.0064 Pas \\
\hline & 7.649 & $0.01 \mathrm{Pas}$ \\
\hline static contact angle, $\theta_{c}$ & \multicolumn{2}{|c|}{$45^{\circ}, 124^{\circ}$} \\
\hline impinging angle $\theta_{\mathrm{i}}$. & \multicolumn{2}{|c|}{$0^{\circ}, 30^{\circ}, 45^{\circ}, 60^{\circ}$} \\
\hline Weber number, $W e=\frac{2 \rho U_{0}^{2} R_{c}}{\sigma}$ & \multicolumn{2}{|c|}{$5.68,22.7$} \\
\hline Ohnesorge number, $O h=\frac{\mu}{\sqrt{2 \rho R_{c} \sigma}}$ & \multicolumn{2}{|c|}{$0.136,0.214$} \\
\hline
\end{tabular}



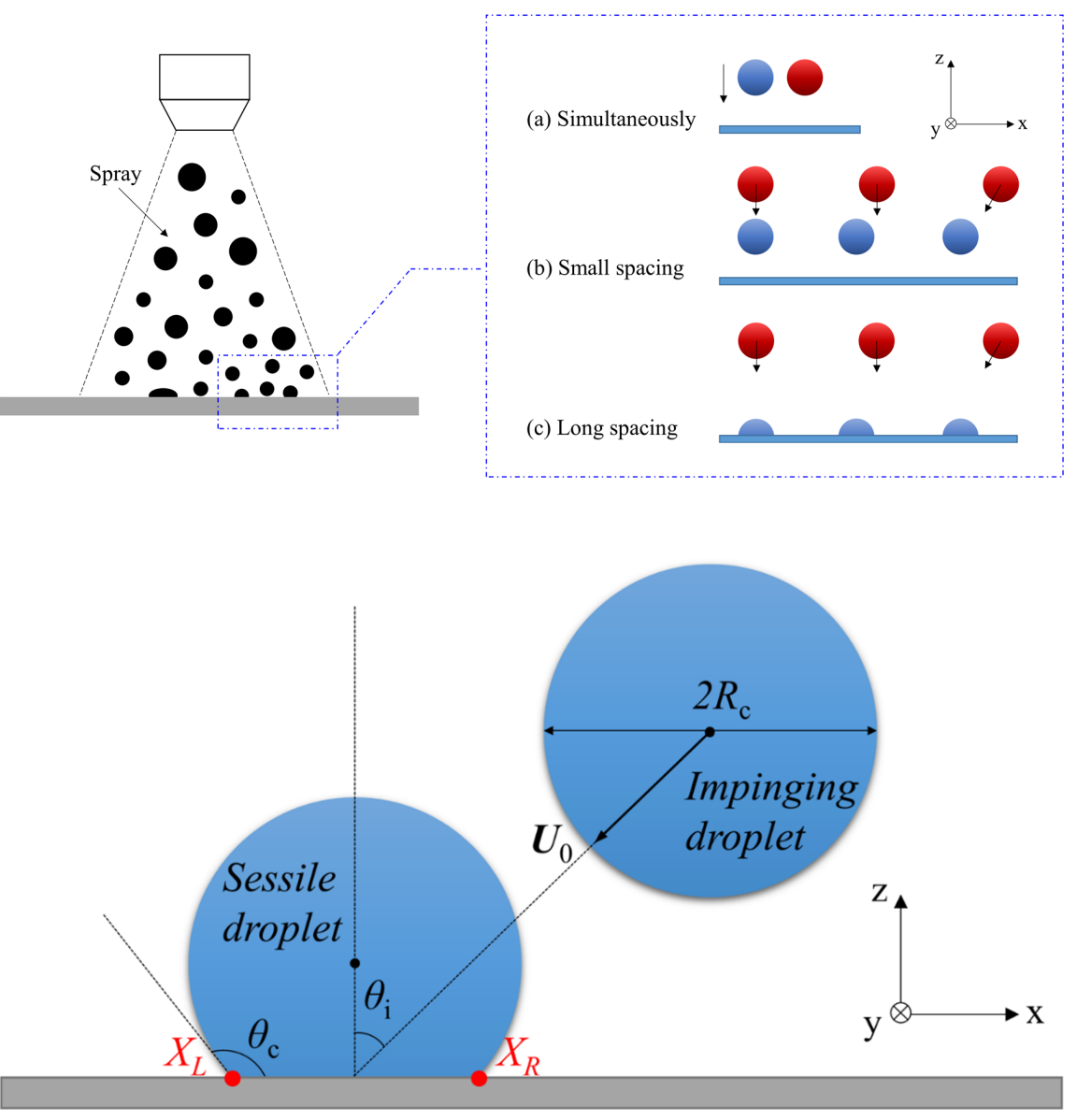

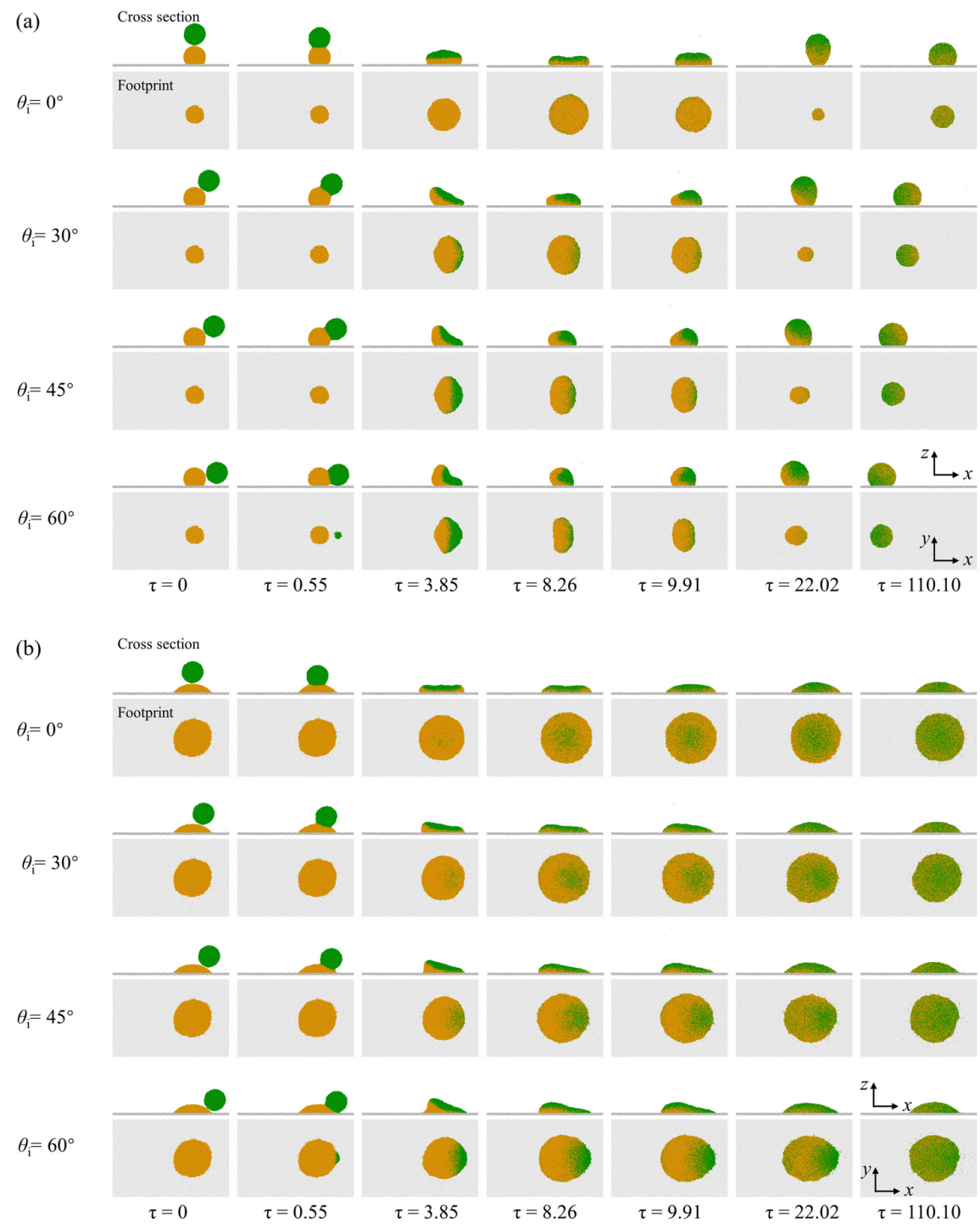

(a)
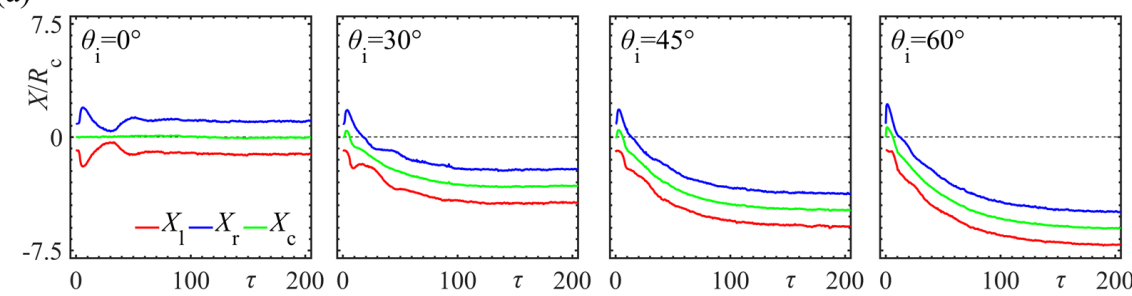

(b)
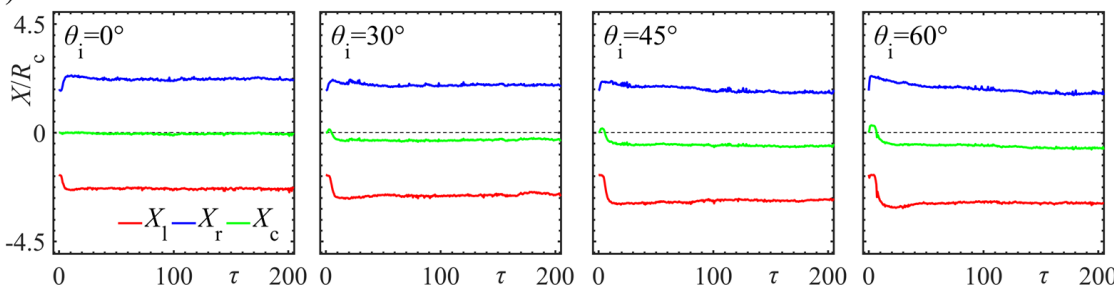

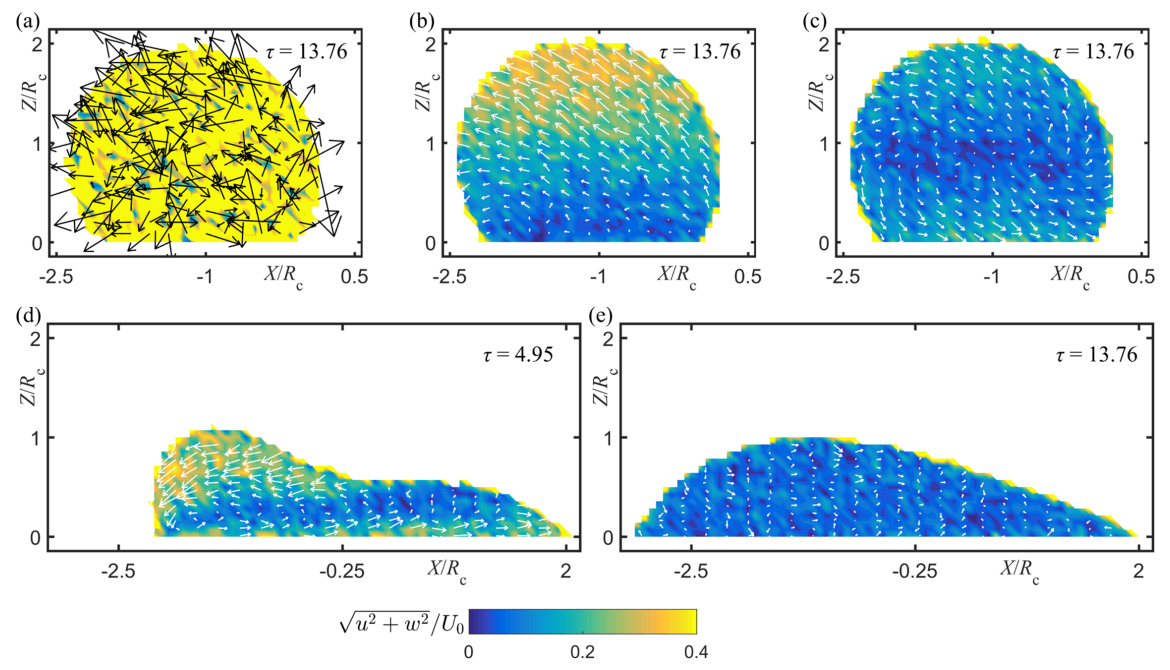

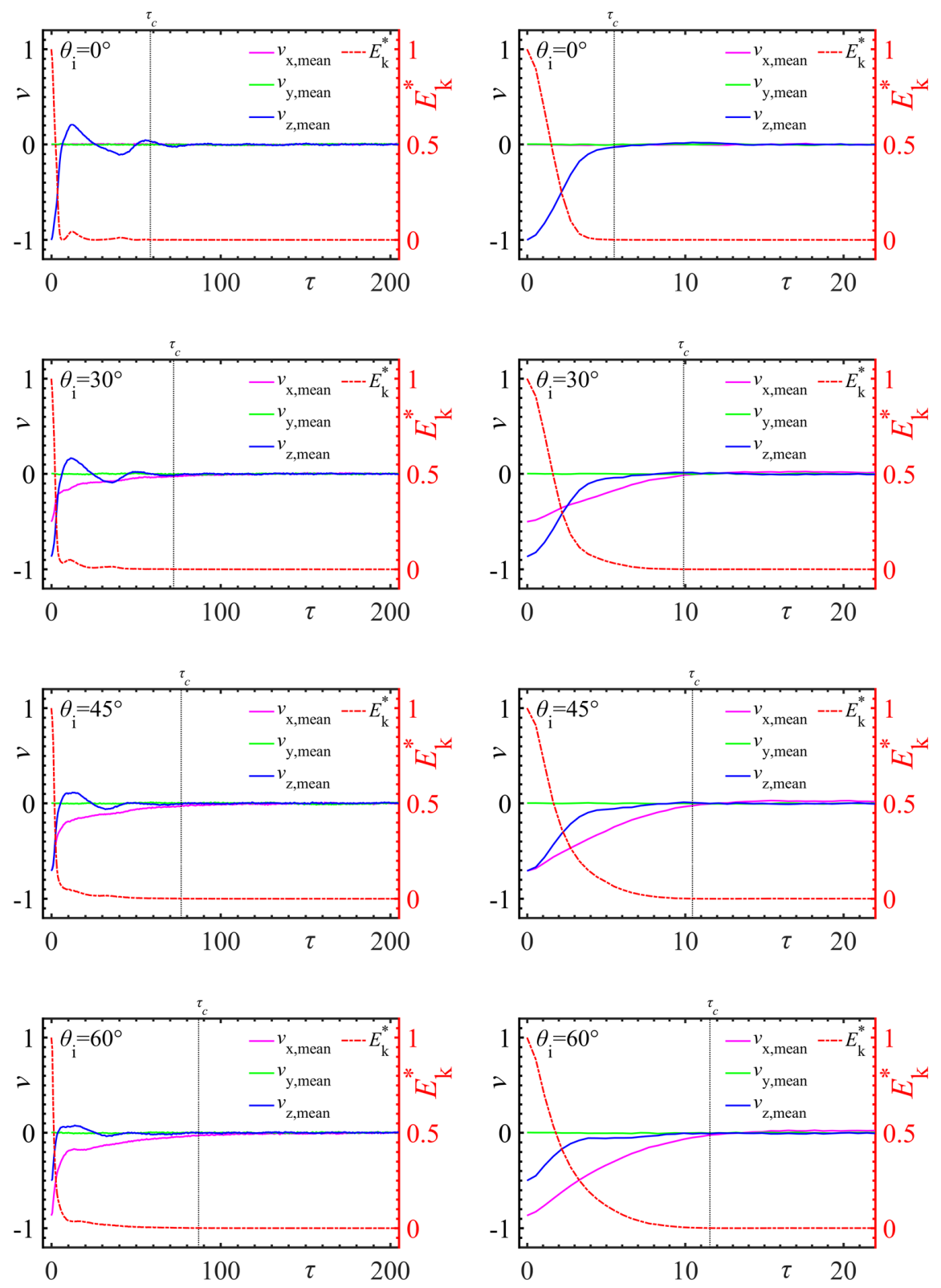
(a)
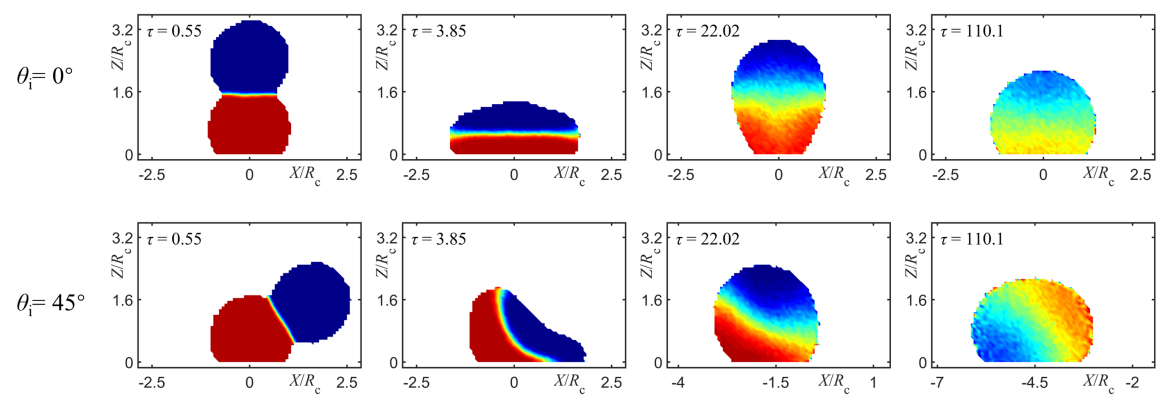

(b)
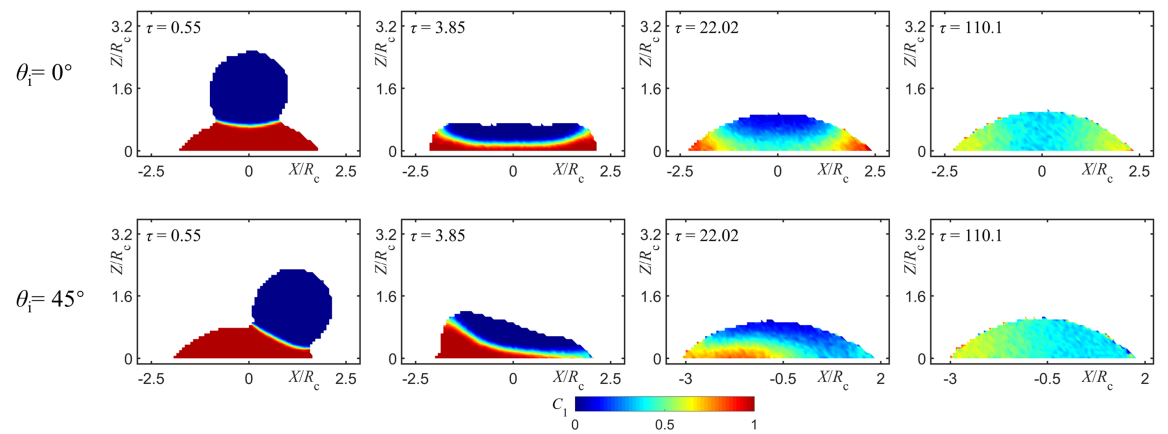

(a)

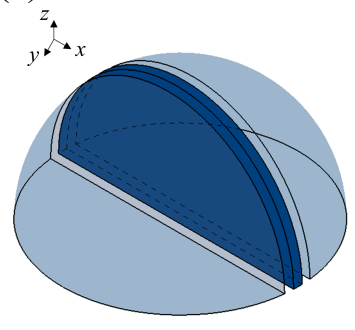

(b)

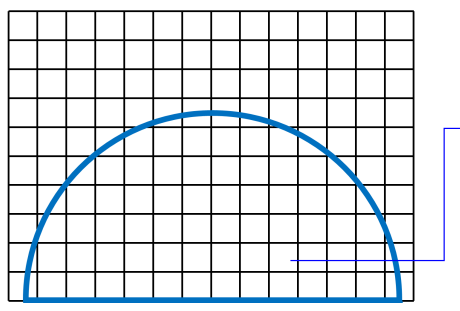

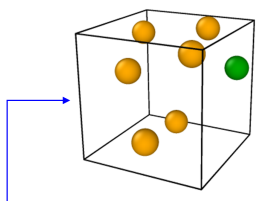

Sessile droplet particle Impinging droplet particle 

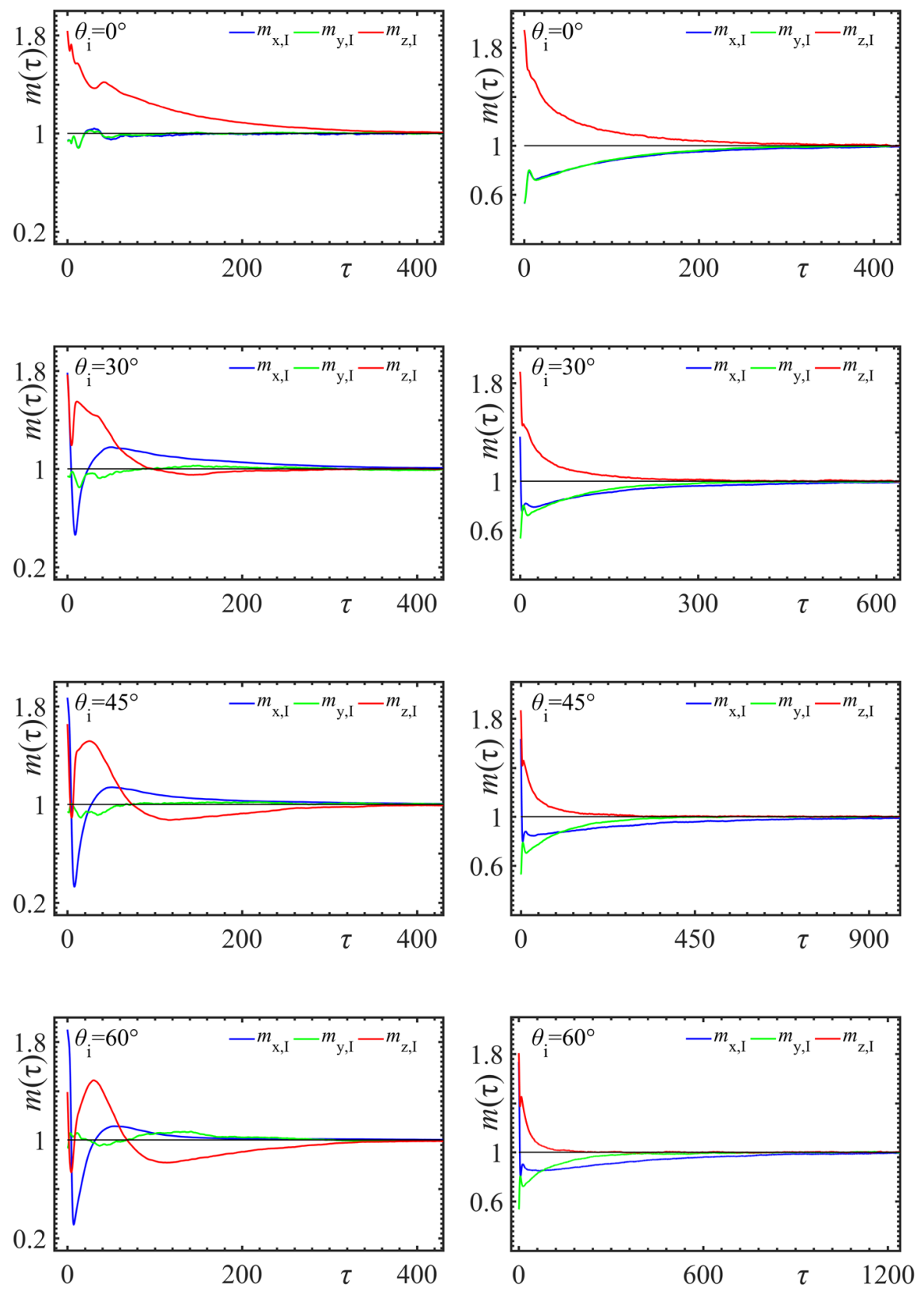

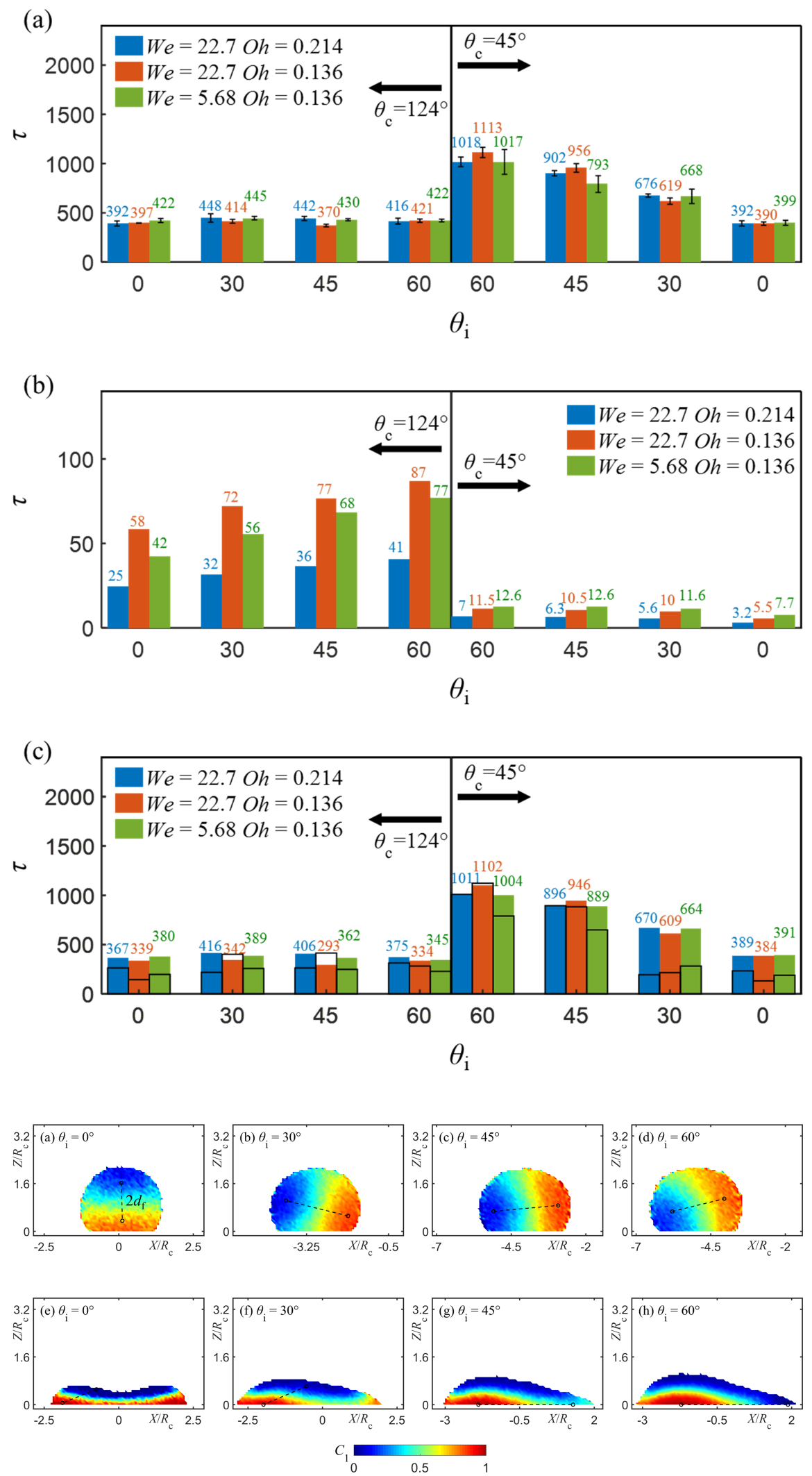


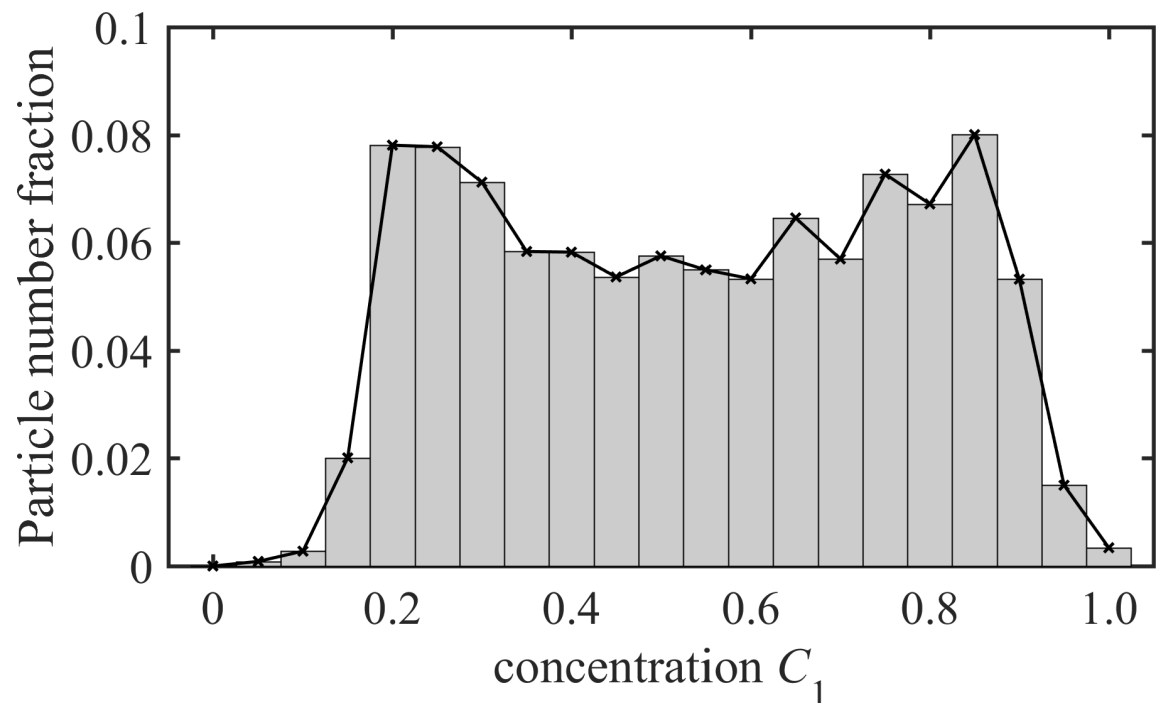

